\title{
Limitations on Electron Beam Density of Unipotential Electron Guns at Low Voltages
}

\author{
J. Arol Simpson and C. E. Kuyatt
}

(Received April 1, 1963)

\begin{abstract}
The problem of saturating a particular configuration of space with electrons is considered. It is shown that with unipotential guns of axial symmetry derived from space charge limited diodes there exist both a minimum energy and a maximum width-to-length ratio of the space that can be saturated. These limits are derived for the case of convergent flow between concentric spheres. Similar limits for partial saturation of the space are derived for cases of convergent, parallel, and divergent flow.
\end{abstract}

One of the constantly recurring problems in electron physics is the generation of low energy electron beams in free space. These beams are usually required to be circular in cross sections of some specified length and diameter and have some energy between 1 and 1,000 ev. The highest electron current possible within these specifications is usually desired. The authors recently gave [1] ${ }^{1}$ a procedure for designing such guns using the multistaging principle, i.e., the use of an intermediate anode operated far above the final beam voltage. Since added complexity and practical difficulties, such as the increased probability of ionizing the residual gas and increased problems of interelectrode leakage, are inherent in such designs, it appears desirable to delineate the regions where the failure of unipotential guns makes multistaging essential.

It is essential to understand the basic physical limitations on achievable electron flow patterns for it is the nature of these limitations which determines the beam profile and density. These limitations are of two classes, one the result of space charge repulsion, the other essentially of thermodynamic nature. The resulting restrictions function both individually and most importantly in combination.

Let us consider the usual case figure 1 . It is desired to irradiate a volume defined by a length $l$ and a diameter $2 r_{0}$. Alternately we may consider the space defined by $l$ and the angle $\gamma$.

As is well known [2], the maximum current, $I_{\max }$, that can be forced through such a space against the dispersive forces of space charge is

$$
I_{\max }=38.5 E^{3 / 2} \frac{2 r_{0}}{l}=38.5 E^{3 / 2} \tan ^{2} \gamma
$$

where current is in microamperes and energies $E$ are in ev. It can be further shown that this results in a maximum current density at the point of minimum cross section (where the beam diameter is $2 r_{0} / 2.35$ ) of

$$
J_{\max }=\frac{211}{\pi r_{0}^{2}} E^{3 / 2} \tan ^{2} \gamma
$$

${ }^{1}$ Figures in brackets indicate the literature references at the end of this paper. where current density is in microamperes per square length unit used in measuring $r_{0}$.

To achieve this maximum the beam must be launched through a hole the same size as the diameter of the space and directed so that in the absence of space charge there would be a crossover in the center of the volume. We note that while the current depends only on the shape but not the size of the space this is not true of the density and moreover no absolute upper limit on density is predicted.

One solution to the problem of generating space charge limited beams and launching them at the proper angle was found by the design techniques developed by Pierce [3] and expanded by Samuel [4], Field [5] and other more recent workers [6, 7].

These solutions consist of a section of a spherical diode where the effects of the radial flow beyond the beam limits are simulated by proper electrode profiles or auxiliary electrodes. When proper account is taken of the lens formed by the hole in the inner sphere (anode lens) such guns can be designed for currents up to the point where the effect of the anode hole on the field at the cathode can no longer be compensated. For guns without an anode grid this point occurs when the hole size approaches the diode spacing or at a point where $I(\mu \mathrm{amp})$ approaches $E^{3 / 2}(\mathrm{v})$, i.e., a microperveance of unity. Particularly useful design curves for such guns may be found in Spangenberg's work [8].

Field [5] seems to have been the first to point out that the effects of the thermal energies of the electrons at the cathode spread the beam and may be an im-

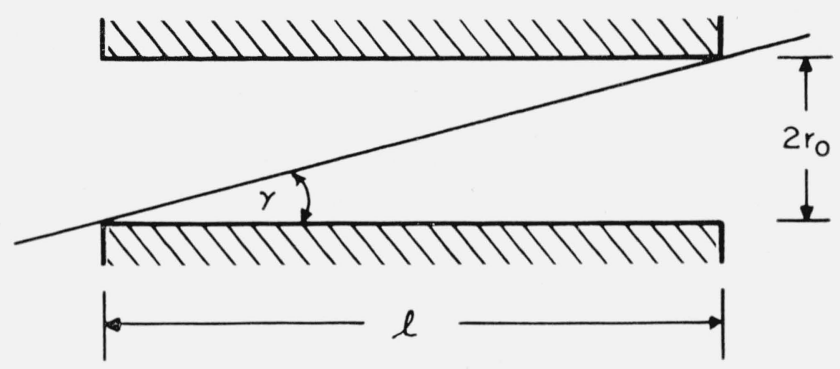

Figure 1. Definition of space to be saturated and of defining angle $\gamma$. 
portant factor in limiting beam current and density. This beam spread is a consequence of a fundamental law of optics which in various forms carries the names of Helmholtz, Lagrange, Clausius, and Langmuir. This law, based upon general arguments of classical mechanics and derivable from Liouville's theorem, states, for electrons, that

$$
E_{1}^{1 / 2} \sin \theta_{1} d \theta_{1} d x_{1}=E_{2}^{1 / 2} \sin \theta_{2} d \theta_{2} d x_{2} .
$$

In this case $E_{1}$ and $E_{2}$ are the energies of the electrons in planes 1 and $2, \theta$ the convergence angles in these planes and $d x_{1}$ and $d x_{2}$ are the corresponding elements of length in these planes.

As written in differential form, this law applies to any system whether or not images are formed. It is generally not very useful since to determine the $x$ 's and $\theta$ 's a complete trajectory calculation must be performed. If, however, planes 1 and 2 are conjugate object and image, or object and crossover then the equation may be integrated to give

$$
E_{1}^{1 / 2} \sin \theta_{1}=M E_{2}^{1 / 2} \sin \theta_{2}
$$

when $M$ is the Gaussian magnification.

If we specialize further to the case where plane 1 is a thermionic cathode and express $E_{1}$ in terms of the cathode temperature and $M$ as a ratio of current densities we can obtain the Langmuir [9] form:

$J_{2}=J_{1}[1+E /(T / 11,600)] \sin ^{2} \theta_{2} \approx J_{1}(11,600 E / T) \sin ^{2} \theta_{2}$.

The general consequences of these equations are well known and are treated at length in the standard texts [10].

If we compare eqs (2) and (3) we see that a priori there is no reason why the density required by eq (2) cannot be obtained subject to eq (3) since $\breve{J}_{1}$ is not limited. In practice of course there are material limits on the cathode, but it can be shown that for energies below a few hundred volts modern cathodes are sufficiently "bright" to satisfy almost any demand.

However, there is a third restriction on unipotential guns which in certain voltage regions prohibits the densities required by eq (2) to saturate a given space. This restriction is the bound on obtainable $J_{1}$ which is set by space charge within the gun structure. Meltzer [11] has shown that a limitation of the form:

$$
J=\frac{k E^{3 / 2}}{S^{2}}
$$

where $S$ a linear dimension is a general characteristic of space charge limited diodes [12]. If we now consider a gun based on such a diode $r_{0}$ can serve to determine the scale and play the role of $S$ in eq (4). The value of the multiplier $k$ is set by the overall geometry necessary to give the convergence angle $\gamma$ in free space beyond the gun. Hence $k$ is a function of $\gamma$. There are, of course, many conceivable guns which give an angle $\gamma$ but for each general class $k$ remains a function of $\gamma$. The result is a limitation on cathode current density of the form:

$$
J_{0}=\frac{f(\gamma) E^{3 / 2}}{r_{0}^{2}} .
$$

Substitution of this value of $J_{0}$ for $J_{1}$ in eq (3) gives the maximum obtainable current density, $J_{2}$, in the space to be saturated. If $J_{2}$ is less than $J_{\max }$ from eq (2), the space cannot be saturated. Combining the equations shows that if

$$
E<\frac{211}{\pi} \frac{T}{11,600} \frac{\tan ^{2} \gamma}{f(\gamma) \sin ^{2} \theta_{2}}
$$

the space cannot be saturated. The connection between $\gamma$, the launching angle, and $\theta_{2}$, the angle at minimum beam density, is somewhat subtle involving as it does the detailed nature of the trajectories between the anode and the point of minimum beam cross section as well as the angle and energy with which a given electron leaves the cathode. However, without this detailed knowledge one fact is clear: $\theta_{2}$ cannot exceed $\gamma$ without some electrons passing outside of the space to be saturated. Hence, we can set $\theta_{2}$ equal to $\gamma$ without violating the inequality.

There is moreover an upper limit on $\gamma$ since the anode hole lens of a diode is always divergent. Hence electrons make a larger angle with the axis just before the lens than they do after the lens. 'Considering a class of guns which give saturation and taking $90^{\circ}$ as a natural limit of the angle inside the lens we see that there is always a maximum value of $\gamma$ for which the space can be saturated. Actually the limit is reached at smaller angles since a large $\gamma$ requires a higher perveance which in most structures requires closer anode cathode spacing resulting in higher fields inside the anode and hence stronger divergence at the anode lens.

For guns based on radial flow between concentric spheres the quantity $f(\gamma)$ in eq (6) may be obtained from the well-known optics of Pierce guns [8]. Pierce [14] has calculated the values of the gun parameters where the space charge spread equals the thermal spread. In our notation this procedue. is equivalent to the assumption that $\gamma$ equals $\theta_{2}$. Danielson, Rosenfeld, and Saloom [6] have done careful numerical calculations on the trajectories in a series of Pierce guns and obtained the final size of the minimum beam cross section when both space charge and thermal spread are important. This procedure, of course, accounts fully for the detailed relationship between $\gamma$ and $\theta_{2}$. Over the range of gun parameters studied, there is no doubt that the latter is the more precise calculation and should be the final check on any proposed design. However, both calculations focus their attention on the gun and require the calculation of a series of guns each of which will saturate the space. It is more useful, especially in the early stages of design of an experiment, to concentrate on the space so that the feasibinity of the experiment may be judged prior to detailed gun design. 
To achieve this end we have evaluated eq (6) using an $f(\gamma)$ derived from the results of Pierce. We assumed a current efficiency of 0.9 and a corresponding intensity efficiency of 0.39. The anode lens was assumed to be of the Davisson [15] form, the $\sim 10$ percent correction of Danielson et al., not being significant for our purposes. We assumed $\gamma$ equal to $\theta_{2}$. Our calculations are hence much in the spirit of those of Pierce but extended to account more fully for the effect of the anode lens. They are somewhat less optimistic than his, but still must be considered outer bounds of possible operation.

The limits found are independent of the scale of the space, depending only on $\gamma$. If the scale is reduced beyond a critical minimum, it is possible that the cathode material limit may be reached. However, if a cathode-anode spacing of $1 \mathrm{~mm}$ is considered a practical lower limit $1 \mathrm{amp} / \mathrm{cm}^{2}$ cannot be exceeded below $265 \mathrm{v}$.

The results of the calculation are shown in figure 2 . On this plot lines of constant perveance, $I / V^{3 / 2}$, appear as straight lines sloping upward to the right. When the space is saturated, as in the upper portion of the graph, eq (1) gives the relation between the perveance and corresponding value of $\gamma$. In this portion of the graph the unity perveance line corresponds to a $\gamma=9.2^{\circ}$. The area to the left of this line is generally not accessible to unipotential guns because of their limited perveance. In the area above the almost horizontal line $I / I_{\max }=1$, spaces characterized by gammas up to $9.2 \mathrm{deg}$ may be fully saturated. Below this $I / I_{\max }=1$ line the spread of the beam due to thermal velocities dominates the situation and frustrates attempts to operate at lower voltages by reducing the current drastically below the value predicted by extending the lines of constant $\gamma$. It will be noted that there is no unipotential gun which will saturate a space below $\sim 220 \mathrm{v}$ beam energy.

If one is prepared to relax the current requirement so that only 0.5 of the maximum possible current is passed, the guns need be less convergent and the area between the perveance $=1.0$ curves now marked $\gamma=12.8$ and $I / I_{\max }=0.5$ is available. The entrance diameter (anode hole of the gun) instead of being equal to the space diameter is now found to equal 0.52 of this diameter.

If one still further reduces the current requirement to $0.25 I_{\max }$ it is possible to use guns whose injection angle into the space is $0^{\circ}$. Under this circumstance the anode hole diameter should be 0.425 of the tube diameter. The details of the calculation are slightly different since now the maximum density lies within the gun structure but the results are of the same form. The minimum usable voltage is decreased to $10 \mathrm{ev}$ and spaces up to $\gamma=17.85^{\circ}$ may be one-quarter saturated.

A sort of natural limit to this process is reached at the dotted curves above and to the right of which the highly divergent parallel diode guns are usable. Although such guns can be used to a minimum of almost 1 ev and to space angles of $\gamma=74^{\circ}$ (we assumed a unity spaced diode of microperveance

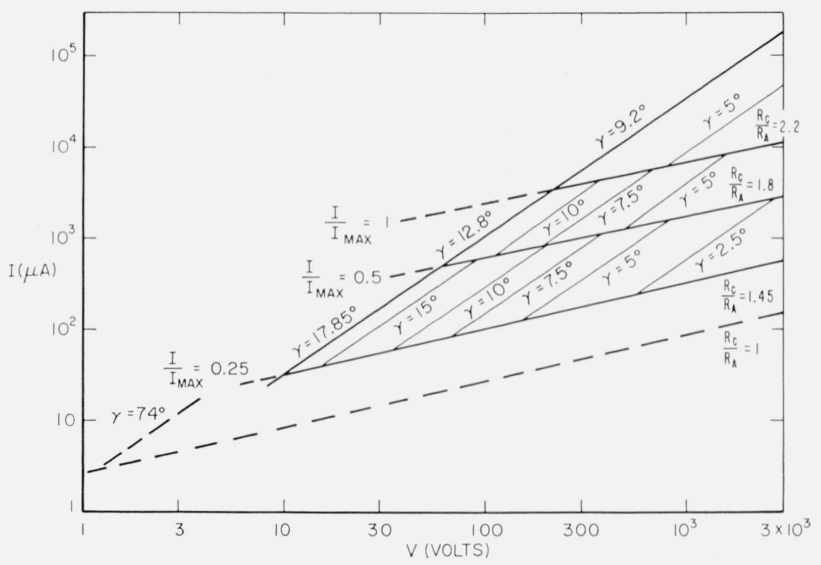

FiguRe 2. Graph of current versus voltage showing regions accessible to guns capable of saturating a given space with $I_{\max }$.

Cases of subsaturation, $0.5 I_{\mathrm{max}}, 0.25 I_{\max }$ and $0.05 I_{\max }$ are shown. The usable range lies to the right and above of all curves. On the right margin are given the ratio of cathode/anode radius of the optimum Pierce gun.

2.33 whose anode diameter is very much smaller than the tube diameter) the fact that they will only put $0.05 I_{\max }$ through any given space makes them not very attractive.

The curves of figure 2 show clearly that the range of applicability of unipotential guns lies at comparatively high energy and wide angles. To operate outside these limits it is necessary to effectively decouple the thermal energy and space charge limit by adopting the multistaging principle and operating a diode or triode at comparatively high voltage and decelerating to the desired low energy.

\section{References}

[1] J. A. Simpson and C. E. Kuyatt, Rev. Sci. Instr. 34, 265 (1963)

[2] E. E. Watson, Phil. Mag. 3, 849 (1927).

[3] J. R. Pierce, J. Appl. Phys. 11, 548 (1940).

[4] A. L. Samuel, Proc. IRE 33, 233 (1945).

[5] L. M. Field, Rev. Mod. Phys. 18, 353 (1946)

[6] W. E. Danielson, J. L. Rosenfeld, and J. A. Saloom, Bell Tech. Jour. 35, 375 (1956).

[7] Müller, Arch. El. Úbertr. 9, 20 (1955).

[8] K. R. Spangenberg, Vacuum Tubes (McGraw-Hill Book Company, Inc., New York, N.Y., 1948).

[9] D. B. Langmuir, Proc. IRE 25, 977 (1937).

[10] J. R. Pierce, Theory and Design of Electron Beams, 2d ed., ch. 8 (Van Nostrand Book Co., Inc., New York,
N.Y., 1954).

[11] B. Meltzer, Proc. Phys. Soc. B62, 431 (1949).

[12] An example of this type of limitation is the well-known Langmuir-Blodgett [13] formula for the current density of a plane parallel diode of electrode spacing $x, \mathrm{~cm}$.

$$
J_{0}=\frac{2.33}{x^{2}} E^{3 / 2}\left(\mu \mathrm{amp} / \mathrm{cm}^{2}\right)
$$

[13] I. Langmuir and K. B. Blodgett, Phys. Rev. 24, 49 (1924).

[14] J. R. Pierce, Theory and Design of Electron Beams, 2d ed., ch. 8 (Van Nostrand Book Co., Inc., New York, N.Y., 1954).

[15] C. J. Davisson and C. J. Calbick, Phys. Rev. 42, 580 (1932).

(Paper 67C4-138) 